\title{
Deutschland-Rente aus Hessen
}

Die hessische Landesregierung hat Ende 2016 einen Vorschlag für eine „Deutschland-Rente" gemacht, um so - endlich - das absinkende Niveau der umlagefinanzierten gesetzlichen Rente flächendeckend durch eine kapitalgedeckte Zusatzrente zu erhöhen. So wie das der Gesetzgeber mit Hilfe der Riesterrente erreichen wollte, es aber nicht erreicht hat. Die Riesterrente wird nicht nur im Hinblick auf Verbraucherschutz, d.h. aufgrund von teilweise sehr hohen Kosten, kritisiert, sondern vor allem auch, weil sie und die betriebliche Altersvorsorge (bAV) nur etwa $70 \%$ der sozialversicherungspflichtig Beschäftigten erreichen. Damit entstehen Sicherungslücken. Und selbst für die $70 \%$, die über die gesetzliche Rente hinaus abgesichert sind, wird das individuelle Absicherungsvolumen oft nicht ausreichen, da viele Betriebsrenten niedrig sind und die meisten Arbeitnehmer den Förderungsrahmen für Riesterrenten nicht ausschöpfen. Der hessische Vorschlag, unterstützt von den Magdeburger Professoren Knabe und Weimann, will eine private Altersvorsorge-Pflicht, wie sie gegenwärtig von Clemens Fuest (ZEW bzw. ifo) ins Spiel gebracht wird, umgehen - und trotzdem das Ziel einer besseren Altersvorsorge erreichen. Der Vorschlag ergänzt das Portfolio der bestehenden Möglichkeiten einer privaten oder betrieblichen Altersvorsorge um eine staatlich organisierte „Standard-Zusatzrente“. Und diese Vorsorge soll allen sozialversicherungspflichtig Beschäftigten als eine Standard-Option angeboten werden. Wer nicht widerspricht (,opt-out“), dessen Arbeitgeber führt den Beitrag an die Krankenkasse ab (die inn weiterleitet) und der Arbeitnehmer erwirbt einen Anspruch. Wer glaubt, dass Zusatzvorsorge nicht notwendig sei oder davon überzeugt ist, dass er selbst besser vorsorgen kann (beispielsweise durch eine Riesterrente bei einer Versicherungsgesellschaft), sagt seinem Arbeitgeber, dass er nicht in die Deutschland-Rente einzahlen soll.

Die Hessen-Idee soll die Vielfalt der Möglichkeiten für freiwillige Altersvorsorge nicht beschneiden, sondern eine zusätzliche Option bieten (wobei kein grundsätzlich neuer „Durchführungsweg“ aufgemacht würde). Die Deutschland-Rente ist sozusagen komplementär zu den Reformen, die die Bundesregierung anstrebt, um die betriebliche Altersversorgung auszubauen - insbesondere in Klein- und Mittelbetrieben. Und in der Tat kann man hoffen: Gäbe es die Deutschland-Rente als eine Option, würde das den Wettbewerb um bessere Vorsorgeprodukte anfachen. Dies könnte nach kurzer Zeit die Riesterrente besser und transparenter als gegenwärtig machen. Und die Tarifparteien hätten einen Anreiz, ihre betriebliche Versorgung zu verbessern. Sie wird durch die Deutschland-Rente auch nicht kannibalisiert, da eine vom Arbeitgeber direkt mitverwaltete bAV zu niedrigeren Kosten möglich ist als die außerhalb von Unternehmen verwaltete Deutschland-Rente. Für Arbeitgeber hätte die DeutschlandRente den Vorteil, dass sie nicht selbst dafür haften. Sie würden sich dadurch keineswegs aus der Finanzierung der Altersvorsorge verabschieden. Denn letztlich hängt die Mitfinanzierung nicht vom Arbeitgeberanteil ab, der an die gesetzliche Rentenversicherung gezahlt wird, sondern von den Tarifabschlüssen!

Für die staatlich organisierte Versicherung, die keine Werbung und Akquise betreiben muss, wären die Verwaltungskosten höchstwahrscheinlich niedrig. Der eigentliche Pfiff der Deutschland-Rente könnte jedoch die Unabhängigkeit von den Renditeschwankungen am Kapitalmarkt sein: Die Rente würde anhand der Durchschnittsverzinsung der vergangenen Jahrzehnte berechnet werden. Wer in einer NiedrigzinsPhase in Rente ginge, müsste keine Mini-Renten befürchten. Der Deutschland-Rente (wie auch den Lebensversicherungen!) müsste vom Gesetzgeber das Recht auf diese

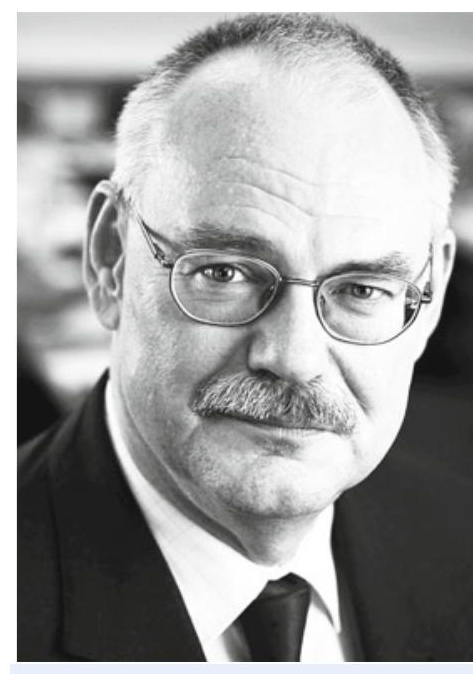

Gert G. Wagner ist Vorstandsmitglied des DIW Berlin, Mitglied im Sachverständigenrat für Verbraucherfragen und Vorsitzender des Sozialbeirats der Bundesregierung. Er äußert hier seine persönliche Meinung. 
„Glättung“ eingeräumt werden - dadurch wäre keine „Beitragsgarantie“ notwendig. Denn zur Not, wenn die Kapitalverzinsung viel schlechter als erwartet läuft, ist eine „Aufstockung“ der umlagefinanzierten gesetzlichen Rente jederzeit möglich. Genau diese Flexibilität zeichnet ja das Umlageverfahren aus. Die für die Riesterrente geltenden Begünstigungen (Subventionen) müssen auch für die Deutschland-Rente gelten. Umgekehrt müssten Riesterverträgen dieselben Bedingungen angeboten werden wie der Deutschland-Rente. Die Einzahlungen für die Riesterrente könnten, um Chancengleichheit zwischen den verschiedenen Renten herzustellen, wie bei der Deutschland-Rente von den Krankenkassen abgebucht werden, die die Beiträge dann weiterleiten. Und Riesterrenten müssten auch ohne Beitragsgarantie angeboten werden dürfen. Diese Garantie ist teuer, da sie festlegt, dass mindestens die vom Versicherten bis zur Fälligkeit des Vertrages eingezahlten Beiträge wieder ausgezahlt werden. Das bindet auch die Versicherer daran, nur in verhältnismäßig sichere Geldanlagen zu investieren. Und darunter leidet die Rendite.

Was auf jeden Fall klar ist: Mehr private Alterssicherung ist im unteren Lohnbereich nur sinnvoll, wenn bei der Grundsicherung im Alter Freibeträge eingeführt werden. Nach geltendem Recht wird die private Rente wie die gesetzliche und betriebliche Rente auf die Grundsicherung angerechnet, wodurch sich für Niedrigverdiener private Vorsorge „nicht rechnet“. Sollte deswegen das Niveau der gesetzlichen Rente entsprechend wieder etwas angehoben werden, um es für Niedrigverdiener einfacher zu machen, durch private Vorsorge, wie durch die Deutschland-Rente, ein Alterseinkommen deutlich über dem Niveau der Grundsicherung zu erzielen? Eine von vielen offenen Fragen. Schließlich, ganz wichtig: Wie sichert man die Unabhängigkeit vom Staat und vom Finanzminister, sodass dieser nicht das angesparte Kapital für andere Zwecke missbrauchen kann? Denkbar wäre eine Einrichtung per (Grund-)Gesetz zu schaffen, die ähnlich unabhängig wie die Bundesbank ist. Im Aufsichtsgremium dieser Institution könnte auch die Bundesbank als politisch unabhängiger Akteur sitzen. Und dem Fonds soll es verboten werden, einen signifikanten Anteil der Aktien eines Unternehmens zu erwerben, um zu verhindern, dass der Staat den Fonds zur Steuerung von Unternehmen missbraucht. Die Sicherung weitgehender Unabhängigkeit ist sicherlich einer der wichtigsten offenen Diskussionspunkte. Dazu gehört auch die Frage, ob die aufwendige Governance von privaten Versicherungen mit allen in der EU erforderlichen Kontrollmechanismen vermieden werden sollte, indem die „Deutschland-Rente“ als Teil der sozialen Sicherung und nicht als private Versicherung gestaltet würde. Die soziale Komponente würde automatisch in den Vordergrund rücken, wenn die Deutschland-Rente auch einer besseren Absicherung der Risiken Berufsunfähigkeit, Erwerbsminderung und Verwitwung dienen würde. Sollten auch Selbständige einbezogen werden? Und sollten diese - sofern sie nicht bereits jetzt speziell abgesichert sind (etwa als Rechtsanwälte) - verpflichtet werden, für sich in der gesetzlichen Rentenversicherung eine Grundabsicherung zu erwerben?

Man kann den Hessen-Vorschlag auch so sehen: Die zentrale Frage ist, ob durch ein Opt-out mehr Menschen dazu gebracht werden sollen, eine kapitalgedeckte Zusatzrente für sich aufzubauen. Wenn diese Frage mit Ja beantwortet wird, ist zu entscheiden, wer eine Standardvorsorge anbietet. Das kann die Deutschland-Rente sein. Es könnten aber auch staatlich zertifizierte private Anbieter sein, denen die Versicherten per Los zugeteilt werden. Die Grundidee der Deutschland-Rente - nämlich die Verbreitung privater Altersvorsorge zu verbessern, muss nicht zwangsläufig mit einer direkt staatlich organisierten Kapitaldeckung realisiert werden. Dies impliziert der Hessen-Vorschlag aber ohnehin nicht: Die Deutschland-Rente soll ja nur eine von

Gert G. Wagner

DIW Berlin

gwagner@diw.de mehreren Optionen sein, die von der privaten Versicherungswirtschaft angeboten werden können. Die Deutschland-Rente soll also - auf Basis eines möglichen Optout - den Wettbewerb befördern. 